\title{
Prevenção de violência e promoção de relações de intimidade saudáveis: Análise interventiva com adolescentes
}

\author{
Preventing violence and promoting healthy intimate relationships: Interventional analysis with \\ adolescents
}

Prevención de la violencia y promoción de relaciones íntimas saludables: Análisis intervencionista con adolescentes

\author{
Marina Soares Souza \\ ORCID: https://orcid.org/0000-0002-9003-6968 \\ Universidade Federal de São Carlos, Brasil \\ E-mail: marinasoaresmss@gmail.com \\ Silvia Mara Carvalho Silva \\ ORCID: https://orcid.org/0000-0003-2847-8383 \\ Universidade Federal de São Carlos, Brasil \\ E-mail: silvinha_smcs_sf@hotmail.com \\ André Vilela Komatsu \\ ORCID: https://orcid.org/0000-0001-8508-6787 \\ Universidade de São Paulo, Brasil \\ E-mail: andevk@gmail.com \\ Angelica Martins de Souza Gonçalves \\ ORCID: https://orcid.org/0000-0002-7265-5837 \\ Universidade Federal de São Carlos, Brasi \\ E-mail: angelicamartins@ufscar.br \\ Natália Rejane Salim \\ ORCID: https://orcid.org/0000-0001-7744-8274 \\ Universidade Federal de São Carlos, Brasil \\ E-mail: nat.salim@gmail.com \\ Diene Monique Carlos \\ ORCID: https://orcid.org/0000-0002-4950-7350 \\ Universidade Federal de São Carlos, Brasil \\ E-mail: dienecarlos@ufscar.br
}

\begin{abstract}
Resumo
Objetivo: avaliar o impacto de um programa de intervenção multicomponente para conscientização de adolescentes sobre violência nas relações íntimas, capacitando-os para a construção de relações saudáveis. Método: Estudo quaseexperimental, com estudantes do $8^{\circ}$ e $9^{\circ}$ ano do Ensino Fundamental de escola pública antes $(\mathrm{n}=105)$, após $(\mathrm{n}=88)$ e após seis meses $(n=82)$ de uma intervenção baseada no modelo ecológico. Os instrumentos de diagnóstico foram o questionário sociodemográfico; de conhecimento sobre violência nas relações de intimidade; e de competências sociais. Utilizou-se estatística descritiva e inferencial, sendo aplicados teste de qui-quadrado e de Wilcoxon. Resultados: A média de idade foi de 13,9 anos; 48,6\% dos adolescentes do sexo feminino; 75,2\% já tiveram algum tipo de relação íntima, onde $6,7 \%$ se reconheceram como vítimas e/ou perpetradores de algum tipo de violência. Os grupos não apresentaram diferenças significativas na comparação dos tempos de coleta. Conclusão: Conclui-se que programas como este ainda necessitam de aprofundamentos práticos, considerando instrumentos de diagnósticos e estratégias selecionadas para intervenções melhor adequadas às necessidades dos participantes a serem alcançados. Ressalta-se a importância de divulgação de experiências não efetivas, visto que resultados negativos podem contribuir para reformulação e aprimoramento para utilizações futuras.

Palavras-chave: Violência por parceiro íntimo; Intervenção; Prevenção primária; Promoção da saúde; Avaliação de programas e projetos de saúde; Adolescente.
\end{abstract}

\begin{abstract}
Objective: to evaluate the impact of a multicomponent intervention program to raise awareness among adolescents about violence in intimate relationships, enabling them to build healthy relationships. Method: Quasi-experimental study with students from the 8th and 9th grade of elementary school in a public school before $(n=105)$, after $(n=88)$ and after six months $(n=82)$ of an intervention based on the ecological model. The diagnostic instruments were the sociodemographic questionnaire; knowledge about violence in intimate relationships; and social skills. Descriptive
\end{abstract}


and inferential statistics were used, using the chi-square and Wilcoxon tests. Results: The average age was 13.9 years; $48.6 \%$ of female adolescents; $75.2 \%$ have already had some type of intimate relationship, where $6.7 \%$ recognized themselves as victims and / or perpetrators of some type of violence. The groups did not present significant differences in the comparison of the collection times. Conclusion: It is concluded that programs like this still need further practical study, considering diagnostic tools and strategies selected for interventions that are better suited to the needs of the participants to be reached. The importance of disseminating ineffective experiences is emphasized, since negative results can contribute to reformulation and improvement for future uses.

Keywords: Intimate partner violence; Intervention; Primary prevention; Health promotion; Program evaluation; Adolescent.

\section{Resumen}

Objetivo: evaluar el impacto de un programa de intervención multicomponente para concienciar a los adolescentes sobre la violencia en las relaciones íntimas, permitiéndoles construir relaciones saludables. Método: Estudio cuasiexperimental con alumnos de $8^{\circ}$ y $9^{\circ}$ de primaria de una escuela pública antes $(\mathrm{n}=105)$, después $(\mathrm{n}=88)$ y después de seis meses $(n=82)$ de una intervención basada en la ecología. modelo. Los instrumentos de diagnóstico fueron el cuestionario sociodemográfico; conocimiento sobre la violencia en las relaciones íntimas; y habilidades sociales. Se utilizó estadística descriptiva e inferencial, utilizando las pruebas de chi-cuadrado y Wilcoxon. Resultados: La edad promedio fue de 13,9 años; 48,6\% de mujeres adolescentes; El 75,2\% ya ha tenido algún tipo de relación íntima, donde el $6,7 \%$ se reconoció como víctima y / o autor de algún tipo de violencia. Los grupos no presentaron diferencias significativas en la comparación de los tiempos de recogida. Conclusión: Se concluye que programas como este aún necesitan un mayor estudio práctico, considerando herramientas de diagnóstico y estrategias seleccionadas para intervenciones que se adapten mejor a las necesidades de los participantes a alcanzar. Se enfatiza la importancia de difundir experiencias ineficaces, ya que los resultados negativos pueden contribuir a la reformulación y mejora para usos futuros.

Palabras clave: Violencia de pareja; Intervención; Prevención primaria; Promoción de la salud; Evaluación de programas y proyectos de salud; Adolescente.

\section{Introdução}

Nos últimos anos, estudos têm se voltado à violência nas relações de intimidade (VRI) entre adolescentes (Minayo et al., 2011; Beserra et al., 2016; Bittar \& Najano, 2017; Carlos et al., 2017; Ferriani et al., 2017). Tal abordagem se faz por alguns fatores, entre eles o estabelecimento precoce de relações de intimidade não saudáveis na vida das pessoas (Centers for disease control and prevention, 2016).

A VRI é considerada como a violência física, sexual, psicológica ou emocional em uma relação de intimidade. Traz a possibilidade de ocorrência de forma pessoal ou eletrônica, através de mensagens de texto repetidas, publicação de fotos íntimas não autorizadas, ou mesmo perseguição, com táticas que provocam medo ao outro. Entre adolescentes é especialmente crítico, considerando que eles possam consentir com estes tipos de comportamentos inadequados por acreditarem ser condição normal de um relacionamento (Centers for disease control and prevention, 2016).

Este fenômeno tem se mostrado comum e transversal a diferentes culturas e etnias. Nos Estados Unidos da América (EUA), o Center for Disease Control and Prevention demonstrou que 26\% das mulheres e 15\% dos homens já sofreram violência física, sexual ou perseguição por parceiro íntimo, vivenciando algum tipo de VRI pela primeira vez antes de completarem 18 anos de idade (Centers for disease control and prevention, 2019). No Brasil, realizou-se um estudo em dez capitais que tratou sobre a violência nas relações de 'ficar' ou namorar entre adolescentes, revelando dados alarmantes. O estudo indicou que $86,9 \%$ já foram vítimas e $86,8 \%$ já praticaram algum tipo de violência, seja física, sexual ou psicológica, durante o relacionamento (Minayo et al., 2011).

A VRI traz consequências à saúde física e mental dos adolescentes, a curto e longo espaço de tempo. Estudos longitudinais demonstraram que adolescentes que são vítimas de VRI apresentam maior risco para sintomas de depressão e ansiedade; envolvimento em comportamentos não saudáveis como uso de tabaco, álcool e outras substâncias psicoativas; aparecimento de comportamentos antissociais e pensamentos suicidas (Foshee et al., 2013; Exner-Cortens et al., 2013). 
Assim, torna-se essencial à agenda da saúde do adolescente a temática de prevenção da VRI precocemente. De acordo com a OMS, mais do que prevenir a violência, reitera-se a necessidade de promover relações interpessoais saudáveis por meio do estímulo às competências sociais e emocionais para resolução de conflitos de forma não violenta, em especial na adolescência (OMS, 2015). A prevenção primária traz ênfase às mudanças nas normas associadas à violência, diminuindo estereótipos de gênero e desenvolvendo habilidades de manejo de conflitos (Foshee et al., 2005). Já a promoção da saúde envolve o processo de tornar as pessoas hábeis a aumentar o controle e promover sua saúde. Desta forma, os profissionais são chamados à implementação de ações nesta vertente, em especial em fenômenos como a violência, com vistas ao empoderamento pessoal (Povlsen \& Borup, 2015).

Os programas de intervenção para prevenção da VRI e promoção de RIS entre adolescentes têm sido amplamente realizados e analisados em países desenvolvidos, em especial nos EUA (Fellmeth et al., 2013; LaRue et al., 2014; Carlos et al., 2017). A literatura segue recomendando intervenções multicomponentes em contexto escolar no âmbito da VRI como prioridade de pesquisa (Fellmeth et al., 2013); uso de desenhos metodológicos mais robustos, garantindo maior nível de evidência cientifica (Lundgren \& Amin, 2015); implementação e avaliação de programas em países em desenvolvimento e subdesenvolvidos (Lundgren \& Amin, 2015). Neste sentido, no contexto brasileiro, são encontrados dois estudos que tratam de intervenções baseadas em habilidades sociais, desenvolvidas em contexto escolar para prevenção de VRI entre adolescentes (Murta et al., 2016; Santos et al., 2019).

Considerando o exposto, a OMS propõe o modelo ecológico para compreensão e prevenção da violência, baseado na evidência de que nenhum fator singular pode explicar o maior risco de algumas pessoas ou grupos à violência interpessoal, enquanto outros são mais protegidos dela. Entende-se este fenômeno como resultado da interação de múltiplos fatores em quatro níveis - individual, relacional, comunitário e social (OMS, 2014).

Assim, este estudo trouxe a seguinte questão de pesquisa: Qual o impacto de uma intervenção multicomponentes, no contexto escolar, para prevenção de VRI e construção de RIS entre adolescentes? As hipóteses levantadas no presente estudo foram: (1) adolescentes participantes da intervenção apresentarão maiores escores nos conhecimentos relacionados à VRI que adolescentes que não participaram da intervenção (grupo-comparação); (2) adolescentes participantes da intervenção apresentarão maiores escores nos parâmetros de competências sociais que podem contribuir para a construção de RIS do que adolescentes que não participaram da intervenção (grupo-comparação).

O objetivo do estudo foi avaliar os efeitos de um programa de intervenção que visa a conscientização de adolescentes sobre a VRI, capacitando-os para a construção de RIS.

\section{Metodologia}

Estudo quase-experimental, com grupo experimental e controle (Shadish et al., 2002), que investigou os efeitos de um programa escolar voltado à promoção e prevenção de relações de intimidade saudáveis entre adolescentes. Para isso, aplicou-se um pré-teste para ambos os grupos, contendo instrumentos que rastrearam o conhecimento sobre VRI e competências sociais que podem promover a construção de RIS entre adolescentes (variáveis dependentes). Em seguida, foi implementada uma intervenção multicomponente sobre relações de intimidade saudáveis (variável dependente) somente para o grupo experimental; após a intervenção aplicou-se um pós-teste com as mesmas variáveis para ambos os grupos, e seis meses reaplicou-se para um follow-up.

Participaram do estudo adolescentes do Ensino Fundamental de uma escola pública estadual de um município do interior de São Paulo, Brasil. Os critérios de inclusão para o grupo experimental e controle foram: ser estudante regularmente matriculado na escola selecionada no $8^{\circ}$ e $9^{\circ}$ ano do ensino fundamental; estar presente nas datas de coleta de dados. Para o 
grupo experimental, foram excluídos os estudantes que não participaram integralmente da intervenção multicomponente.

Para a composição do grupo experimental foi feito um sorteio para cada uma das turmas as demais turmas representaram, então, o grupo-comparação, alocados conforme Figura 1:

Figura 1. Fluxograma de recrutamento dos participantes.

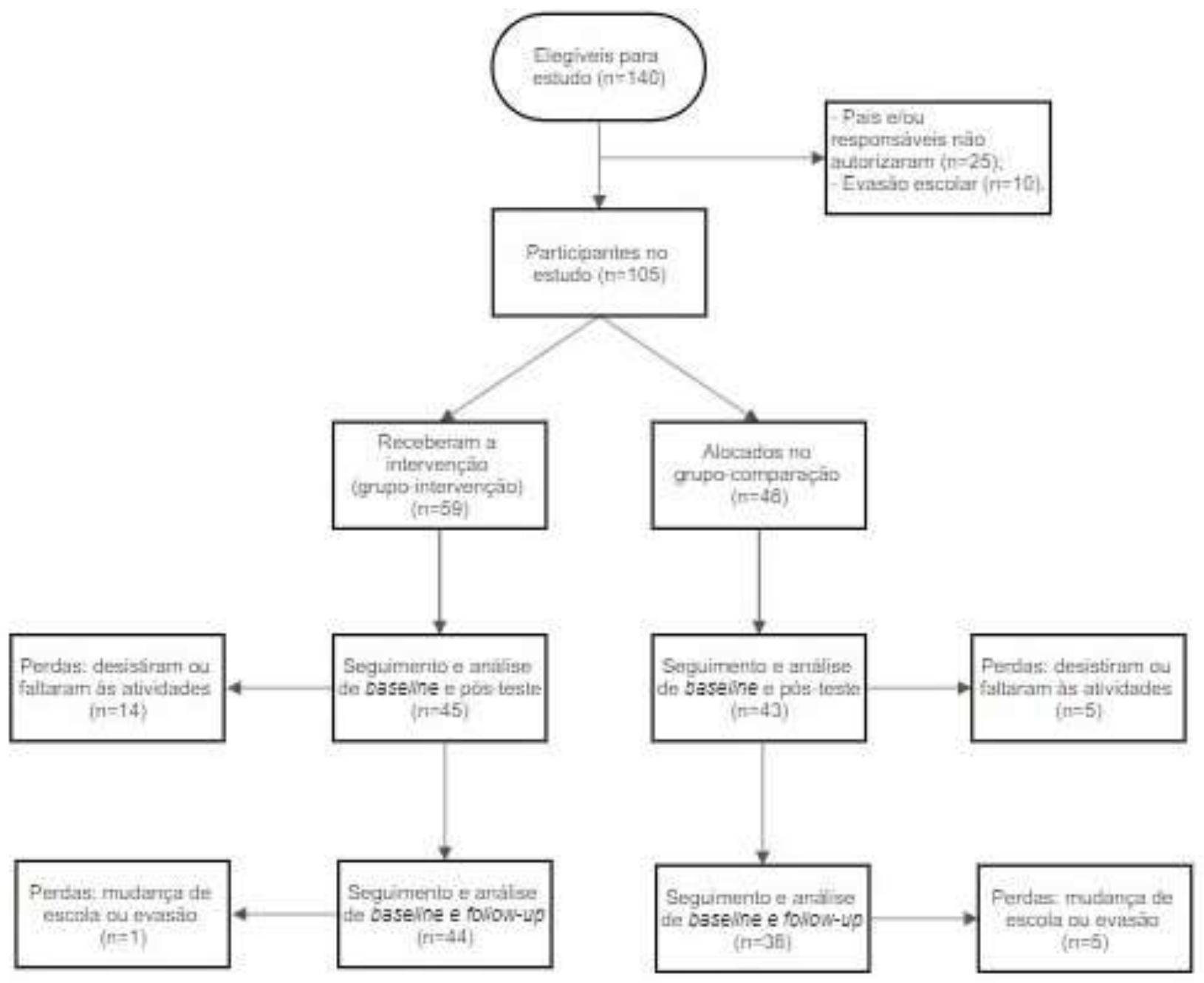

Fonte: Elaboração própria, adaptado (CONSORT 2010).

A composição dos grupos levou em conta a semelhança quanto à idade, ano letivo e condições socioeconômicas. O estudo foi conduzido em local de alta vulnerabilidade, Grupo 5 de acordo com o Índice Paulista de Vulnerabilidade Social (Brasil, 2019).

A coleta de dados ocorreu entre maio e dezembro de 2019. A intervenção foi aplicada por duas pesquisadoras treinadas e deu-se em quatro sessões, de 50 minutos. Os componentes da intervenção tiveram objetivos específicos para abordar o tema de violência nas relações de intimidade e para alcançá-los, utilizaram-se diferentes estratégias, conforme apresentado na Tabela 1: 
Tabela 1. Componentes da intervenção, segundo sessões, objetivos e estratégias, São Paulo, Brasil, 2019

\begin{tabular}{|c|c|c|}
\hline Sessão & Objetivos & Estratégia \\
\hline 1 & $\begin{array}{l}\text { - Apresentar a intervenção; } \\
\text {-Levantar os interesses/expectativas dos } \\
\text { estudantes; } \\
\text { - Sensibilizar para a VRI. }\end{array}$ & $\begin{array}{l}\text { - Teatro sobre situação hipotética de VRI; } \\
\text { - Exposição dialogada; } \\
\text { - Check-list sobre consequências da VRI; } \\
\text { - Mapa mínimo da rede pessoal/social. }\end{array}$ \\
\hline 2 & $\begin{array}{l}\text { - Conceituar: papéis de gênero e estereótipos; } \\
\text { - Discutir as implicações de gênero na saúde; } \\
\text { - Tratar igualdades / desigualdades de gênero; } \\
\text { direitos humanos. }\end{array}$ & $\begin{array}{l}\text { - Dinâmica "Age como homem/Age com mulher"; } \\
\text { - Diálogo sobre o gênero como uma construção social e } \\
\text { sobre como os estereótipos refletem na saúde; } \\
\text { - Jogo "“"No que diferem os homens e as mulheres?". }\end{array}$ \\
\hline 3 & $\begin{array}{l}\text { - Retomar questões da violência (conceito, } \\
\text { ciclo, fatores de risco e proteção); } \\
\text {-Abordar sobre Comunicação Não Violenta } \\
(\mathrm{CNV})\end{array}$ & $\begin{array}{l}\text { - Brainstorming sobre os principais pontos da violência } \\
\text { trabalhados anteriormente, } \\
\text {-Atividades "Descobrindo como me sinto" e "Desenho às } \\
\text { cegas!" } \\
\text { - Vídeo sobre CNV. }\end{array}$ \\
\hline 4 & - Discutir elementos para a construção de RIS & $\begin{array}{l}\text { - Oficinas pedagógicas: "Receita de bolo para um bom } \\
\text { relacionamento!" e "Construindo árvores/relações"; } \\
\text { - Avaliação final. }\end{array}$ \\
\hline
\end{tabular}

Fonte: Elaborado pelos autores (2021)

O conteúdo programático da intervenção pautou-se no referencial teórico do modelo ecológico, trazendo a discussão de fatores de risco e proteção para a VRI, bem como elementos para promover relações saudáveis (OMS, 2014). A execução do estudo ocorreu em horários e espaços cedidos pela direção da escola por favorecer a adesão e envolvimento dos participantes, como também por permitir a construção de um vínculo entre os participantes e pesquisadores.

O instrumento aplicado nos três tempos consistiu de: (1) questionário de caracterização sociodemográfica (sexo, idade e constituição familiar) e das relações de intimidade (se já namorou/namora, idade de início das relações, número de pessoas que se relacionou, relações sexuais e quanto à perpetração ou vitimização de VRI); (2) Questionário de Conhecimento sobre Violência nas Relações de Intimidade (QCVRI), baseado em Leitão e colaboradores (2013), em que o conhecimento é uma variável qualitativa nominal, correspondendo aos conhecimentos que os participantes detém sobre VRI; (3) Questionário de Competências Sociais (QCS), traduzido para o português por Mota, Matos e Lemos (2011). A versão traduzida utilizada neste estudo apresentou consistência interna moderada com valores de alfa de Cronbach de 0.87 , considerando o instrumento válido e fiável para investigação em estudos.

A pesquisa foi aprovada pelo Comitê de Ética em Pesquisa da instituição universitária ao qual os pesquisadores são vinculados, sob o parecer $n^{\circ}$ 3.215.561/ 2019, respeitando os critérios da Resolução do Conselho Nacional de Saúde $n^{\circ}$ 466/2012 e n510/2016. O estudo foi autorizado pela direção da instituição de ensino e encaminhado o TCLE aos pais dos adolescentes. Todos os adolescentes foram informados quanto aos objetivos desse estudo, sendo-lhes requisitada assinatura do TALE para participação.

Os dados coletados foram inseridos em uma planilha eletrônica e analisados com o software R. Foram computados as médias e os desvios-padrão dos grupos Controle e Intervenção em cada período de tempo - pré, pós e follow-up - e para cada instrumento aplicado - QCVRI e QCS. As pontuações dos grupos foram comparadas por meio do teste de Wilcoxon para amostras dependentes e o valor de $\mathrm{p}$ foi ajustado pelo método de Bonferroni para lidar com o viés de múltiplas comparações.

\section{Resultados}

Os adolescentes apresentaram média de idade de 13,9 anos com idade mínima de 13 anos e máxima de 16 anos. Observou-se maioria de sexo feminino (48,6\%). Quanto à característica familiar os maiores percentuais foram referentes ao residir com a mãe $(87,6 \%)$ e irmãos $(76,2 \%)$. No que se refere ao número de pessoas que moram na mesma casa, o mais 
sinalizado pelos adolescentes foi de 5 pessoas $(25,7 \%)$. Quanto à escolaridade dos responsáveis, observou-se maiores percentuais para o Ensino Médio incompleto (21\% para mães; 13,3\% para pais).

Os grupos (intervenção e comparação) foram equivalentes em relação às variáveis de relacionamento íntimo, não apresentando diferenças estatisticamente significativas para idade de início das relações íntimas ( $\underline{x}=11,34 ; 11,05$ respectivamente). A média de pessoas que os participantes já se relacionaram foi um pouco maior no grupo intervenção $(8,61)$ em relação ao grupo comparação $(8,15)$. A média de idade da primeira relação sexual foi de 12 anos para o grupo intervenção e de 13,33 anos para o grupo comparação. Para perpetração e vitimização de VRI, os resultados indicaram que o grupo comparação sofreu e praticou mais violência, apresentando $2 \%$ de perpetração e $2 \%$ de vitimização para cada tipo de violência indicada: física, psicológica e sexual, tendo o grupo intervenção apresentado $1 \%$ para perpetração de violência sexual e $1 \%$ para vitimização de violência sexual. Entretanto, não houve diferenças estatisticamente significativas na comparação entre os grupos.

As médias e desvios-padrão obtidos no pré e pós-teste e no período de follow-up, em cada questionário, de ambos os grupos está apresentada na Tabela 2:

Tabela 2. Comparação dos grupos em relação aos tempos de coleta (pré-teste, pós-teste e follow-up), apresentando média e desvio dos instrumentos de diagnóstico, São Carlos, São Paulo, Brasil, 2019.

\begin{tabular}{lcccccc}
\hline Instrumento & \multicolumn{2}{c}{ Pré-teste } & \multicolumn{2}{c}{ Pós-teste } & \multicolumn{2}{c}{ Follow-up } \\
Grupo & $\begin{array}{c}\text { Intervenção } \\
(\mathbf{n = 5 9 )} \\
\text { Média (DP) }\end{array}$ & $\begin{array}{c}\text { Controle } \\
(\mathbf{n = 4 6 )} \\
\text { Média (DP) }\end{array}$ & $\begin{array}{c}\text { Grupo } \\
\text { Intervenção } \\
(\mathbf{n = 4 5 )} \\
\text { Média (DP) }\end{array}$ & $\begin{array}{c}\text { Grupo } \\
\text { Controle } \\
(\mathbf{n = 4 3 )} \\
\text { Média (DP) }\end{array}$ & $\begin{array}{c}\text { Grupo } \\
\text { Intervenção } \\
(\mathbf{n}=\mathbf{4 4}) \\
\text { Média (DP) }\end{array}$ & $\begin{array}{c}\text { Grupo } \\
\text { Controle } \\
(\mathbf{n = 3 8}) \\
\text { Média } \\
(\mathbf{D P})\end{array}$ \\
\hline QCVRIvr & $20,2(4,82)$ & $19,6(6,57)$ & $23,2(8,86)$ & $21,5(6,88)$ & $22,7(3,5)$ & $21,1(4,1)$ \\
QCS.Freq & $41,5(10,97)$ & $41,1(13,06)$ & $46,1(16,51)$ & $44,0(16,10)$ & $46,5(13,2)$ & $43,3(10,5)$ \\
QCS.Imp & $40,2(9,59)$ & $41,5(9,68)$ & $45,1(14,15)$ & $43,1(15,71)$ & $45,3(7,3)$ & $43,4(8,5)$ \\
\hline
\end{tabular}

Fonte: Elaborado pelos autores (2021).

$\mathrm{O}$ teste de Wilcoxon não identificou nenhuma diferença significativa $(\mathrm{p}>0.05)$ entre os grupos Controle $\mathrm{e}$ Intervenção em nenhum dos questionários aplicados.

\section{Discussão}

Os resultados apresentados permitiram responder à questão de pesquisa, a saber: "Qual o impacto de uma intervenção multicomponentes para prevenção de VRI e construção de RIS entre adolescentes?", uma vez que não foi observado variação significativa quanto ao nível de conhecimento sobre VRI, como também se verificou variação não significativa sobre as competências sociais dos adolescentes. Assim, as duas hipóteses criadas foram refutadas.

Estes resultados corroboram o achado de estudo americano, que investigou 1517 adolescentes em quatro escolas, encontrando resultados não significativos para a identificação de comportamentos preditivos de VRI após intervenção multicomponentes (Miller et al., 2015). Os autores sugeriram a possibilidade de a taxa de reconhecimento da violência ser menor no início da adolescência, uma vez que tiveram como público-alvo adolescentes com média de 12 a 13 anos de idade. Discutiram, ainda, sobre a recorrência de comportamentos relevantes a exemplo de "cutucadas" ou empurrões como uma forma dos adolescentes demonstrarem interesse por iniciar uma relação de intimidade com algum parceiro, indicando que esses comportamentos podem subestimar o discernimento da VRI, tornando-os mais tolerantes a situações violentas (Miller et al., 2015). 
Assim, a discussão sobre a habilidade de reconhecimento da VRI entre adolescentes deve considerar o estágio de desenvolvimento social e emocional do adolescente. Fator este que também pode contribuir para a não identificação dos sinais de alerta em relacionamentos, como confundir comportamentos controladores e possessivos com aspectos de "amor verdadeiro" (Miller, 2017). Além desta questão, a experiência de relações íntimas pode colaborar em maior reconhecimento de situações violentas. Um estudo transversal epidemiológico que investigou sobre a prevalência da VRI entre adolescentes de 14 a 19 anos associada a comportamentos violentos, observou que quanto maior a idade e o tempo da relação de intimidade entre os adolescentes, maiores eram as chances de percepção de vitimização ou perpetração da violência (Beserra et al., 2016). Tal aspecto pode ser observado ainda em estudo transversal que buscou identificar os fatores que influenciam o reconhecimento da VRI e assertividade sexual em adolescentes mais velhos, de 18 a 20 anos, na Coréia do Sul (Kim, Lee, \& Lee, 2019). Nas análises, o reconhecimento da VRI foi mais expressivo em adolescentes que tinham maior experiência do que os que tinham pouca ou nenhuma experiência. Estes aspectos devem ser considerados de forma mais profunda em intervenções junto a adolescentes, podendo gerar diferenças mais significativas que as apresentadas neste estudo.

Quanto às competências sociais, o estudo em tela apresentou diferenças nas médias em ambos os grupos, ainda que não significativas estatisticamente. Diferentemente, os programas que apresentaram mudanças comportamentais positivas foram realizados em maior tempo de duração, com sessões de 50 minutos a 2 horas, num período de 21 sessões anuais a três anos (STAR; The Youth Relationships Project; The Fourth R) (Saavedra \& Machado, 2013). Este pode ser um dos pontos que justifique as diferenças obtidas neste estudo em relação às demais produções que tiveram mudanças positivas, visto que tais mudanças requerem um tempo maior de intervenção.

Em avaliação do programa de prevenção de VRI Dat-e Adolescence, na Espanha, encontraram-se resultados semelhantes. O programa não observou eficácia quanto à mudança no comportamento dos adolescentes de 11 a 19 anos participantes do estudo. Embora o grupo intervenção tenha apresentado mudança na pontuação, o resultado não foi suficiente para detectar diferenças significativas entre o grupo comparação. Os autores também sugeriram que é necessário um tempo maior para que o programa possa refletir melhoras na aprendizagem e consolidação de competências e estratégias saudáveis que permitem a resolução de conflitos de forma não violenta, reduzindo comportamentos violentos (Sánchez-Jiménez, MuñozFernández, \& Ortega-Rivera, 2018).

No programa Me \& You nos EUA com adolescentes de 11 a 14 anos, os participantes foram avaliados quanto à exposição à VRI no baseline e no segundo follow-up, indicado no estudo como seguimento de doze meses da intervenção (Peskin et al., 2019). No primeiro follow-up - cerca de dois meses após a intervenção, os autores indicaram não ter questionado sobre perpetração e vitimização da VRI, por acreditarem ser tempo insuficiente para mudança de comportamento. Do grupo que já tinham experimentado relações de intimidade, não houve fatores psicossociais estatisticamente significativos no primeiro follow-up, no entanto, no seguimento de doze meses, alguns resultados revelaram significância estatística. Dentre os fatores psicossociais, os autores assumem o enfrentamento à violência, autoeficácia para resolução de conflitos, habilidades de comunicação e maior rede de apoio (Peskin et al., 2019).

Outra avaliação de programa de prevenção de VRI com abordagem de competências sociais foi o Teen Choices, que ocorreu em vinte escolas dos EUA, encontrando melhora significativa no uso de competências sociais saudáveis em relações de intimidade de adolescentes no Ensino Médio (Levesque et al., 2016). O estudo sustentou os resultados significativos em seis e doze meses de seguimento da avaliação do programa. As autoras discutiram ainda a importância da participação dos professores na condução do programa. Enunciaram possibilidades de os professores conduzirem discussões em classe, no intuito de explorar e reforçar conceitos de competências sociais e comportamentos para construção de relacionamentos saudáveis, incluindo atividades para casa ou mesmo em classe, com as quais os adolescentes pudessem escrever sobre o que aprenderam nas sessões discutidas (Levesque et al., 2016). Em nosso estudo, focaram-se os estudantes. No entanto, a literatura 
reforça que esses atores, por terem um contato diário e direto com os adolescentes, tornam-se fundamentais para a prevenção e para o reconhecimento de casos de VRI, uma vez que podem comparar comportamentos em tempos e entre potenciais vítimas ou perpetradoras com seus pares (Donat et al., 2016).

Ainda pensando nas intervenções multicomponentes, o modelo ecológico traz à discussão da inclusão não só de professores nos esforços preventivos da VRI entre adolescentes, mas também de seus pais e responsáveis. Em revisão integrativa, analisou-se 30 produções científicas sobre prevenção de VRI entre adolescentes, considerando gênero e geração (Oliveira et al., 2016). Dentre as produções, duas também incluíram as famílias como objeto de pesquisa. O estudo sinalizou que durante o acompanhamento dos familiares notou-se maior entendimento da gravidade da VRI entre adolescentes, aumento de resposta eficaz para prevenção de VRI e maior abertura ao diálogo com adolescentes sobre o assunto, mostrando-se potente para continuidade de discussão da temática e na comunicação entre responsáveis e adolescentes (Oliveira et al., 2016).

Em relação ao nível comunitário, proposto pelo modelo ecológico, estudo demonstrou que áreas menos favorecidas geralmente estão mais expostas a fatores de risco da VRI, podendo ver a violência com uma resposta esperada, tolerada ou necessária (Garthe et al., 2018). O presente estudo foi desenvolvido em área de alta vulnerabilidade social, que pode estar exposta a maiores fatores de risco mencionados anteriormente. Intervenções que mobilizem e atuem sobre níveis comunitários são viáveis e podem ser um diferencial para efeitos mais significativos na prevenção da VRI e promoção de RIS.

Das limitações plausíveis deste estudo, pode se considerar a não existência de instrumentos de diagnóstico para os adolescentes mais jovens. Em estudo com adolescentes de 15 a 17 anos de escola pública de Brasília-DF foram avaliados com alto grau de instrução, além de pertencerem a uma região com poder aquisitivo elevado (Murta et al., 2016). Os resultados da intervenção multicomponentes foram favoráveis ao reconhecimento da violência; ao desenvolvimento de competências sociais; pensamento crítico acerca de papéis de gênero; direitos sexuais e reprodutivos; e protagonismo do adolescente (Murta et al., 2016). Tal disparidade pode indicar uma inadequação dos instrumentos de diagnóstico para os adolescentes da pesquisa em tela. Pesquisadores já avaliaram a qualidade dos questionários direcionados às investigações de VRI, considerando os instrumentos originais e as adaptações nas línguas inglesa, francesa, portuguesa e espanhola (Yanéz-Peñúñuri et al., 2019). Os autores sugeriram que por se tratar de formas de investigação de prevenção da VRI em idade precoce, é importante atentar para a interpretabilidade e sensibilidade desses questionários, para além de outras propriedades de medida (Yanéz-Peñúñuri et al., 2019).

Além da limitação apresentada acima, também se pontua a fragilidade quanto à execução ou implementação das intervenções, referindo-se ao conteúdo, procedimentos e qualidade na relação dos facilitadores e adolescentes (Murta et al., 2016) Neste estudo, todas as sessões foram avaliadas qualitativamente pelos adolescentes. Todavia, medidas de autoobservação, mensurando o desempenho dos facilitadores, com análises específicas dos resultados obtidos quanto ao papel do moderador, se fazem importantes para investigações futuras. Além destes aspectos, o número de sessões com adolescentes pode ter sido insuficiente, bem como a não intervenções em contextos comunitários mais amplos, considerando o modelo ecológico, podem ser elencados como limitações.

\section{Conclusões}

O objetivo desse estudo foi avaliar um programa de intervenção que visa a conscientização de adolescentes sobre VRI, capacitando-os para construção de RIS. Evidenciou-se que os adolescentes participantes do programa não apresentaram diferenças significativas nos conhecimentos sobre VRI e competências sociais para a construção de RIS após a intervenção.

Apesar dos resultados demonstrados, reforça-se o pioneirismo do estudo na temática, na área da Enfermagem, em contexto de país em desenvolvimento e considerando a abordagem ecológica da violência. Destaca-se a importância de 
fortalecer os pilares de um programa de intervenção multicomponentes para prevenção de VRI e construção de RIS, no que diz respeito ao contexto e adequação do conteúdo e procedimentos utilizados nas abordagens para uma maior aceitação do programa por participantes em potencial. Neste sentido, este estudo traz subsídios para futuras intervenções, além da necessidade de novos estudos com desenhos metodológicos que garantam maior período para follow-up, bem como mais adequados a adolescentes mais jovens. Ademais, a aplicabilidade em outros contextos se faz urgente. Ressalta-se a importância de divulgação de experiências não efetivas, visto que resultados negativos podem contribuir para reformulação e aprimoramento para utilizações futuras.

\section{Agradecimentos}

Apoio Financeiro do Conselho Nacional de Desenvolvimento Científico e Tecnológico (CNPq), Projeto Universal Processo 233534/2014-8, Brasil.

\section{Referências}

Beserra, M. A., Leitão, M. N. D. C., Fabião, J. A. D. S. A. D. O., Dixe, M. D. A. C. R., Veríssimo, C. M. F., \& Ferriani, M. D. G. C. (2016). Prevalence and characteristics of dating violence among school-aged adolescents in Portugal. Escola Anna Nery, 20(1), 183-191.

Bittar, D. B., \& Nakano, A. M. S. (2018). Symbolic violence among adolescents in affective dating relationships. Revista da Escola de Enfermagem da USP, 51 .

Brasil (2019). Fundação Sedae. Índice Paulista de Vulnerabilidade Social: Distribuição da População, segundo Grupos do Índice Paulista de Vulnerabilidade Social. http://www.iprs.seade.gov.br/ipvs2010/view/index.php

Carlos, D. M., Campeiz, A. B., da Silva, J. L., Fernandes, M. I. D., da Cruz Leitão, M. N., Silva, M. A. I., \& Ferriani, M. D. G. C. (2017). School-based interventions for teen dating violence prevention: integrative literature review. Revista de Enfermagem Referência, 4(14), $133-143$.

Centers for disease control and prevention (CDC) (2019). Center for Injury Prevention and Control, Division of Violence Prevention. Preventing Teen Dating Violence. Fact Sheet: National https://www.cdc.gov/violenceprevention/pdf/tdv-factsheet.pdf

Centers for disease control and prevention (CDC) (2016). National Center for Injury Prevention and Control, Division of Violence Prevention. Understanding Teen Dating Violence. Fact Sheet. https://www.cdph.ca.gov/Programs/CCDPHP/DCDIC/SACB/CDPH\%20Document\%20Library/Teen\%20Dating\%2 0Violence/TeenDatingViolenceFactsheet2016.pdf

Donat, J., De Moura, T. C., Nuñez Carvalho, J. D. C., \& Kristensen, C. H. (2016). Professores e maus-tratos-uma revisão teórica sobre reconhecimento, denúncia e capacitação. Revista Educação (PUCRS. Online), 39(1), 66-73.

Exner-Cortens, D., Eckenrode, J., \& Rothman, E. (2013). Longitudinal associations between teen dating violence victimization and adverse health outcomes. Pediatrics, 131(1), 71-78.

Fellmeth, G. L., Heffernan, C., Nurse, J., Habibula, S., \& Sethi, D. (2013). Educational and Skills-Based Interventions for Preventing Relationship and Dating Violence in Adolescents and Young Adults: A Systematic Review. Campbell Systematic Reviews, 9(1), i-124.

Ferriani, M. D. G. C., Campeiz, A. B., Martins, J. E., Aragão, A. D. S., Roque, E. M. D. S. T., \& Carlos, D. M. (2019). Understanding and contextualizing teen dating violence. Escola Anna Nery, 23(3).

Foshee, V. A., Bauman, K. E., Ennett, S. T., Suchindran, C., Benefield, T., \& Linder, G. F. (2005). Assessing the effects of the dating violence prevention program "Safe Dates" using random coefficientregression modeling. Prevention Science, 6(3), 245-258.

Foshee, V. A., Reyes, H. L. M., Gottfredson, N. C., Chang, L. Y., \& Ennett, S. T. (2013). A longitudinal examination of psychological, behavioral, academic, and relationship consequences of dating abuse victimization among a primarily rural sample of adolescents. Journal of Adolescent Health, 53(6), 723-729.

Garthe, R. C., Gorman-Smith, D., Gregory, J., \& E. Schoeny, M. (2018). Neighborhood concentrated disadvantage and dating violence among urban adolescents: The mediating role of neighborhood social processes. American journal of community psychology, 61(3-4), 310-320.

Kim, Y., Lee, E., \& Lee, H. (2019). Sexual double standard, dating violence recognition, and sexual assertiveness among university students in South Korea. Asian nursing research, 13(1), 47-52.

De La Rue, L., Polanin, J. R., Espelage, D. L., \& Pigott, T. D. (2014). School-based interventions to reduce dating and sexual violence: A systematic review. Campbell Systematic Reviews, 10(1), 1-110.

Leitão, M., Fernandes, M. I., Fabião, J. S., Sá, M. C., Veríssimo, C. M., \& Dixe, M. A. (2013). Prevenir a violência no namoro: N (Amor)O (Im)Perfeito: Fazer diferente para fazer a diferença. Monografia. Coimbra, Portugal: Escola Superior de Enfermagem de Coimbra.

Levesque, D. A., Johnson, J. L., Welch, C. A., Prochaska, J. M., \& Paiva, A. L. (2016). Teen dating violence prevention: Cluster-randomized trial of Teen Choices, an online, stage-based program for healthy, nonviolent relationships. Psychology of violence, 6(3), 421. 
Lundgren, R., \& Amin, A. (2015). Addressing intimate partner violence and sexual violence among adolescents: emerging evidence of effectiveness. Journal of Adolescent Health, 56(1), S42-S50.

Miller, E. (2017). Prevention of and interventions for dating and sexual violence in adolescence. Pediatric Clinics, 64(2), 423-434.

Miller, S., Williams, J., Cutbush, S., Gibbs, D., Clinton-Sherrod, M., \& Jones, S. (2015). Evaluation of the Start Strong initiative: preventing teen dating violence and promoting healthy relationships among middle school students. Journal of adolescent health, 56(2), S14-S19.

Minayo, M. C. D. S., Assis, S. G. D., \& Njaine, K. (2011). Amor e violência: um paradoxo das relações de namoro e do 'ficar'entre jovens brasileiros. Editora Fiocruz.

Mota, C. P., Matos, P. M., \& Lemos, M. S. (2011). Psychometric properties of the social skills questionnaire: Portuguese adaptation of the student form (grades 7 to 12). The Spanish Journal of Psychology, 14(1), 486-499.

Murta, S. G., Moore, R. A., Miranda, A. A. V., Cangussú, E. D. A., Santos, K. B. D., Bezerra, K. L. T., \& Veras, L. G. (2016). Efeitos de um programa de prevenção à violência no namoro. Psico-USF, 21(1), 381-393.

Oliveira, Q. B. M., Assis, S. G. D., Njaine, K., \& Pires, T. D. O. (2017). Violência física perpetrada por ciúmes no namoro de adolescentes: Um recorte de gênero em dez capitais brasileiras. Psicologia: teoria e pesquisa, 32(3).

Organização Mundial da Saúde (OMS) (2015). Fapesp. Prevenindo a violência juvenil: um panorama das evidências. https://apps.who.int/iris/bitstream/handle/10665/181008/9789241509251-por.pdf?sequence=5

Organização Mundial da Saúde (OMS) (2014). Violence Prevention Alliance (VPA). Definition and typology of violence. http://www.who.int/violenceprevention/approach/definition/en/index.html

Peskin, M. F., Markham, C. M., Shegog, R., Baumler, E. R., Addy, R. C., Temple, J. R., ... \& Tortolero Emery, S. R. (2019). Adolescent dating violence prevention program for early adolescents: The Me \& You randomized controlled trial, 2014-2015. American journal of public health, 109(10), 1419-1428.

Povlsen, L., \& Borup, I. (2015). Health Promotion: A developing focus area over the years. Scandinavian journal of public health, 43(16_suppl), 46-50.

Saavedra, R., \& Machado, C. (2012). Programas de prevenção primária da violência nos relacionamentos íntimos: uma revisão sistemática da prática internacional e portuguesa. Violência nas relações juvenis de intimidade. Braga: Psiquilíbrios Edições.

Sánchez-Jiménez, V., Muñoz-Fernández, N., \& Ortega-Rivera, J. (2018). Efficacy evaluation of" Dat-e Adolescence": A dating violence prevention program in Spain. PLoS One, 13(10).

Santos, K. B. D., Murta, S. G., Vinha, L. G. D. A., \& Deus, J. S. D. (2019). Efficacy of a bystander intervention for preventing dating violence in Brazilian adolescents: short-term evaluation. Psicologia: Reflexão e Crítica, 32(20).

Shadish W. R., Cook T. D., Campbell, D. T. (2002). Experimental and quasi-experimental designs for generalized causal inference. Boston: Houghton Mifflin.

Yanez-Peñúñuri, L. Y., Hidalgo-Rasmussen, C. A., \& Chávez-Flores, Y. V. (2019). Systematic review of dating violence questionnaires in Ibero-America and evaluation of their measurement properties. Ciencia \& saude coletiva, 24, 2249-2262. 